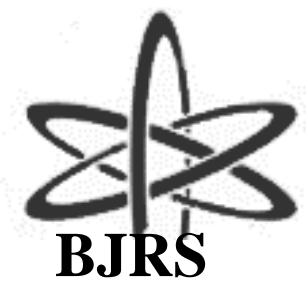

\author{
BRAZILIAN JOURNAL \\ $\mathrm{OF}$ \\ RADIATION SCIENCES \\ 06-02-B (2018) 01-11
}

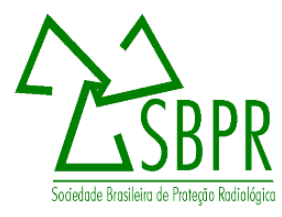

\title{
Realização do teste end-to-end para a caracterização de um simulador em radioterapia estereotática de fígado
}

\author{
A. F. Burgos ${ }^{\text {a }}$ L. P. da Silva ${ }^{\text {b }}$ E. de Paiva ${ }^{a}$ \\ ${ }^{a}$ Divisão de Física Médica, Instituto de Radioproteção e Dosimetria (IRD/CNEN), \\ 22783-127, Rio de Janeiro-RJ, Brasil \\ ${ }^{b}$ Departamento de Física Médica, Instituto Nacional de Câncer (INCA/MS), \\ 20230-130, Rio de Janeiro-RJ, Brasil \\ adamfburgos@gmail.com
}

\section{RESUMO}

Atualmente, por conta do seu bom prognóstico, uma das alternativas ao tratamento radioterápico do fígado é a radioterapia estereotática corpórea (SBRT), que entrega altas doses ao volume alvo em poucas frações. No entanto, para que se assegure que o elevado valor de dose entregue ao alvo seja o mesmo que o planejado, um teste de verificação de todo o processo (aquisição de imagens, delineamento, planejamento e a entrega da dose) deve ser realizado. Para isto, o objetivo deste trabalho foi desenvolver um simulador, com densidade de água, que leva em conta a posição relativa do fígado e dos órgãos de risco envolvidos neste tratamento, avaliando a influência da movimentação do alvo, em virtude do processo respiratório, no valor da dose por ele recebida. Também foram avaliados os valores de dose nas posições relativas aos órgãos de risco.

Palavras-chave: SBRT, figado, efeito interplay, dosimetria. 


\begin{abstract}
Nowadays, one of the alternatives to the radiotherapy treatment of the liver is the stereotactic body radiation therapy (SBRT), which delivers high doses to the target volume in a few fractions. However, in order to ensure that the high dose value delivered to the target is the same as planned, a full process verification known as test end-to-end (image acquisition, design, planning, and dose delivery) should be performed. The aim of this work was to develop a water density simulator that takes into account the relative position of the liver and the risk organs involved in this treatment, evaluating the influence of the movement of the target, due to the respiratory process, in the value of the dose received by the target. The dose values were also evaluated in the positions related to the risk organs.
\end{abstract}

Keywords: SBRT, liver, interplay effect, dosimetry.

\title{
1. INTRODUÇÃO
}

A radioterapia estereotática corpórea (SBRT) de fígado é caracterizada por apresentar um elevado nível de precisão na entrega da dose. No entanto, a elevada dose de radiação por fração utilizada em SBRT requer um elevado nível de precisão nos cálculos de dose de modo a assegurar a entrega da dose prescrita no volume alvo, enquanto se poupa o tecido normal adjacente. Aliado a isto, é fundamental que haja um controle da qualidade da dosimetria que atenda adequadamente os protocolos internacionais vigentes [1-4]. O tratamento do câncer de fígado com SBRT apresenta certos níveis de toxicidade no fígado, e nos órgãos de risco adjacentes (medula óssea e rins). Com isto, torna-se fundamental estabelecer um alto valor de precisão, não só nos processos clínicos, mas também no controle da qualidade do acelerador linear destinado à realização deste tipo de tratamento. Para tal, é necessário que haja um controle dosimétrico preciso e mais próximo da realidade, fazendo-se o uso de certas ferramentas, que promovam essas condições. A elaboração de um simulador físico humano (do inglês, phantom), que leve em conta a forma e a posição relativa dos órgãos supracitados, possibilita uma medição mais próxima da realidade sobre o processo de interação da radiação utilizado nos protocolos clínicos de SBRT de fígado. Além disso, deve-se levar em conta a influência da movimentação do fígado, por conta do processo respiratório, assim como já é conside- 
rado nos protocolos clínicos de SBRT de fígado. Com isto, torna-se recomendável a simulação dos efeitos da radiação, em um simulador especializado, de modo que o alvo do tratamento em questão (fígado) possa ser analisado em uma dosimetria física, que leve em conta a influência da movimentação do mesmo, de acordo com o processo respiratório.

\section{MATERIAIS E MÉTODOS}

Foi desenvolvido um simulador de formato cilíndrico, preenchível com água, com entradas para câmara de ionização, dosímetros termoluminescentes (TLDs) e filme radiocrômico. Esse simulador foi tomografado, com a colocação de marcadores radiopacos. A escolha do formato do fantoma foi baseada em uma forma similar à curvatura da região abdominal de um corpo humano e que exclua a dependência angular. Para tal, o formato cilíndrico foi o escolhido. Com isto, uma estrutura de raio constante (cilindro), reduz a influência desta dependência angular resultando em um registro mais preciso da dose, em cada uma das estruturas analisadas.

O material utilizado para a confecção da estrutura deste simulador, foi o polimetilmetacrilato (PMMA), cuja densidade de $1,18 \mathrm{~g} / \mathrm{cm}^{3}$ se aproxima da densidade média do corpo humano. A estrutura do cilindro é oca, de modo que somente as superfícies do mesmo são constituídas de PMMA, e seu interior é vazio, com o intuito de preenchê-lo com água (Figura 1). A escolha desta estrutura se baseou na densidade próxima da água ( $\rho_{\text {água }}=0,987 \mathrm{~g} / \mathrm{cm}^{3}$, à $20^{\circ} \mathrm{C}$ ), em virtude da semelhança com a densidade dos órgãos que serão analisados (fígado, rins e medula espinhal). 
Figura 1: Simulador preenchido com água (parte superior - à esquerda ; parte inferior - à direita)

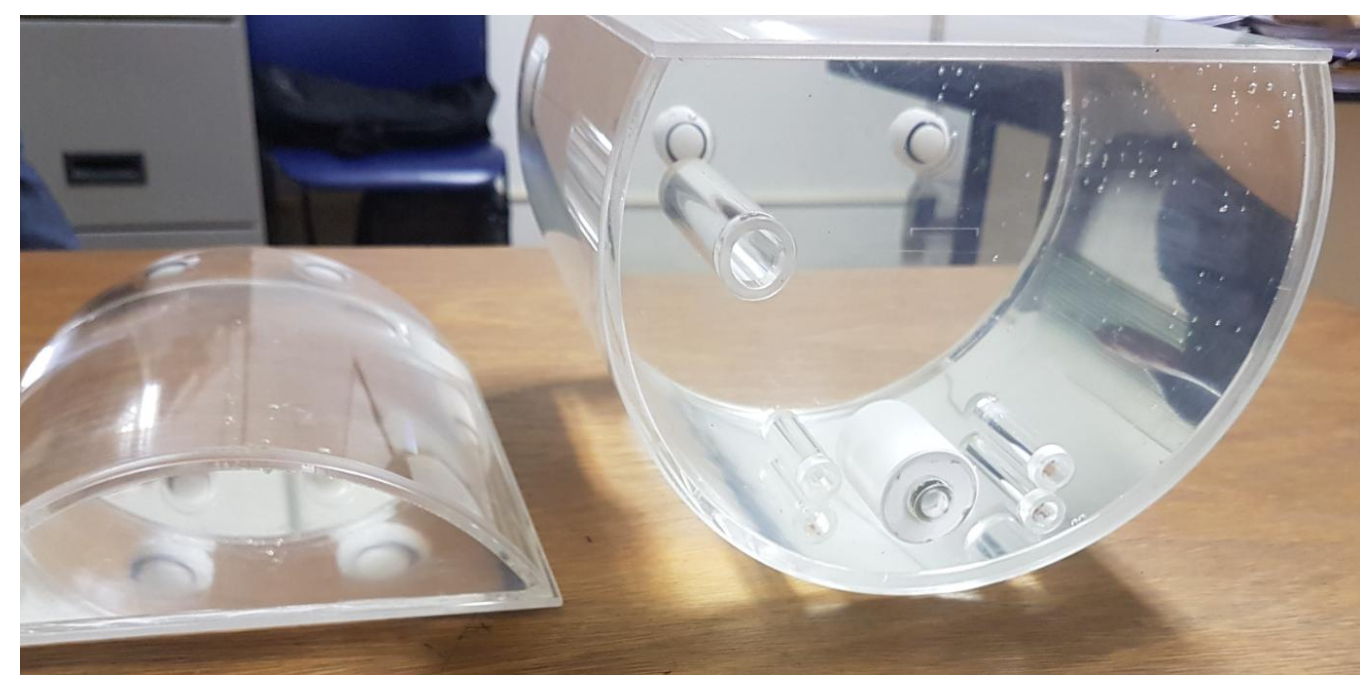

Cada uma das entradas (do inglês, inserts) foi confeccionada para a colocação dos dispositivos de dosimetria física (detectores), com o intuito de determinar a dose absorvida representativa de cada órgão específico (Figura 2).

Figura 2: Função de cada insert do simulador

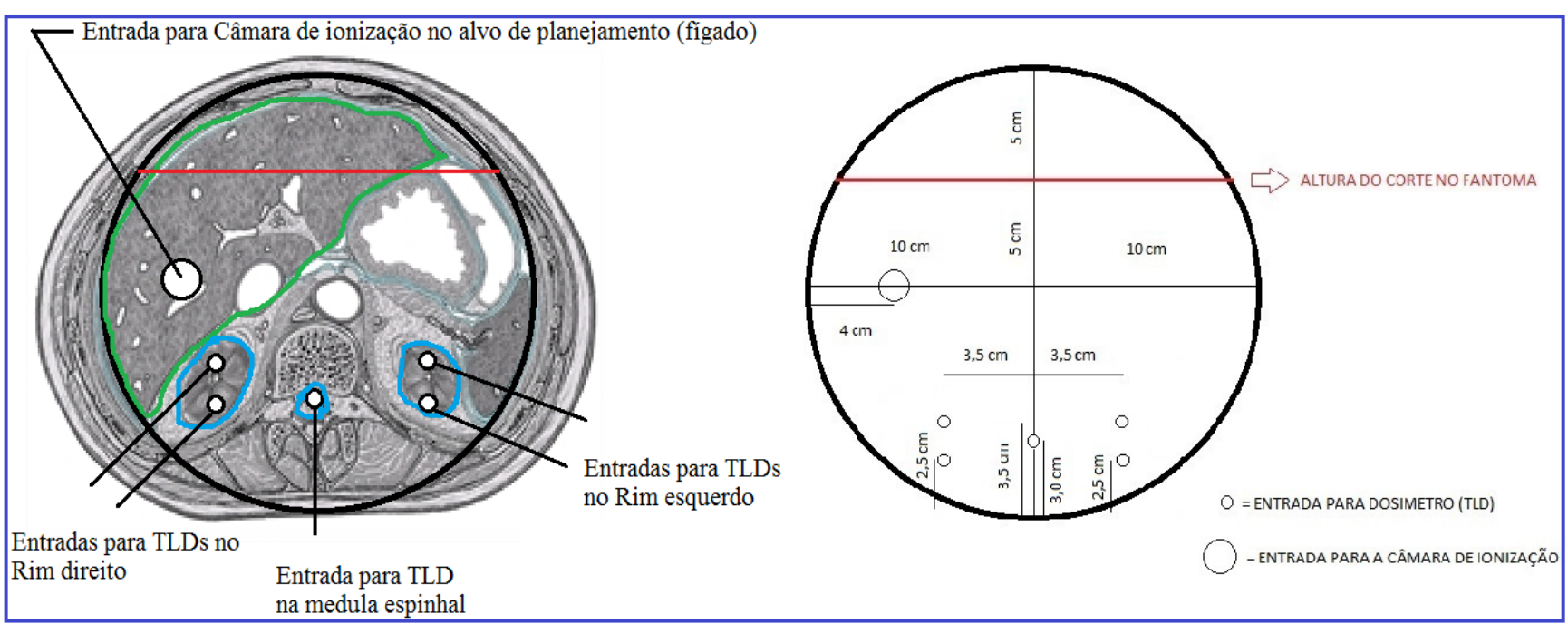


Os TLDs utilizados (LiF:Mg,Ti - TLD 100 Harshall) foram calibrados através da irradiação de parte de um mesmo lote de dosímetros, em uma única irradiação, com um irradiador de Cobalto60 (Theratron 780C) de taxa de dose conhecida, em um intervalo de tempo conhecido para determinar o valor de dose absorvida, que neste caso foi de 600 cGy. O conjunto de dosímetros irradiados foi posicionado dentro de um campo de irradiação $(5 \mathrm{~cm}$ x $5 \mathrm{~cm})$, entre duas placas de água sólida de $5 \mathrm{~cm}$ de espessura, com a placa situada abaixo dos TLD utilizada para posicionar os mesmos dentro do campo luminoso em uma superfície nivelada, e a placa situada acima para simular uma interação da radiação com o corpo humano. Foram separados dosímetros nãoirradiados do mesmo lote para análise da radiação de fundo (background-BG). A partir destes dois conjuntos de dosímetros, foram obtidos os valores das leituras através de uma leitora específica para TLD em pó (PCL3 - Fimel). A incerteza desses valores está associada à leitora de TLDs, uma vez que o desvanecimento e a correção por linearidade não foram necessários em virtude do intervalo de tempo entre a irradiação e a leitura ter sido menor do que o recomendado pelo fabricante e pela faixa de dose utilizada estar dentro da faixa linear de resposta para o tipo de TLD utilizado. Os TLDs foram inseridos nas posições relativas dos órgãos de risco, acondicionados em um recipiente de formato cilíndrico. A câmara de ionização foi inserida em outro insert, em duas diferentes posições representando o deslocamento do alvo (lesão) nos pontos máximos de inspiração e expiração, com o intuito de se gerar uma margem de movimentação do alvo, representado por um volume de tratamento denominado Internal Target Volume (ITV). O ITV é um volume de tratamento que leva em conta uma margem além da massa tumoral, de alterações microscópicas subclínicas, e da movimentação da massa tumoral, que na análise deste estudo, está associado ao processo de inspiração e expiração. Um dispositivo, que executa um movimento periódico para simular o processo respiratório, foi utilizado para movimentar a câmara de ionização (Figura 3). A medida do deslocamento foi realizada com uma régua milimetrada. A incerteza associada à medição do deslocamento corresponde à metade da menor medida da régua milimetrada, que é de 0,5 mm. 
Figura 3: Visão lateral do dispositivo, acionado manualmente

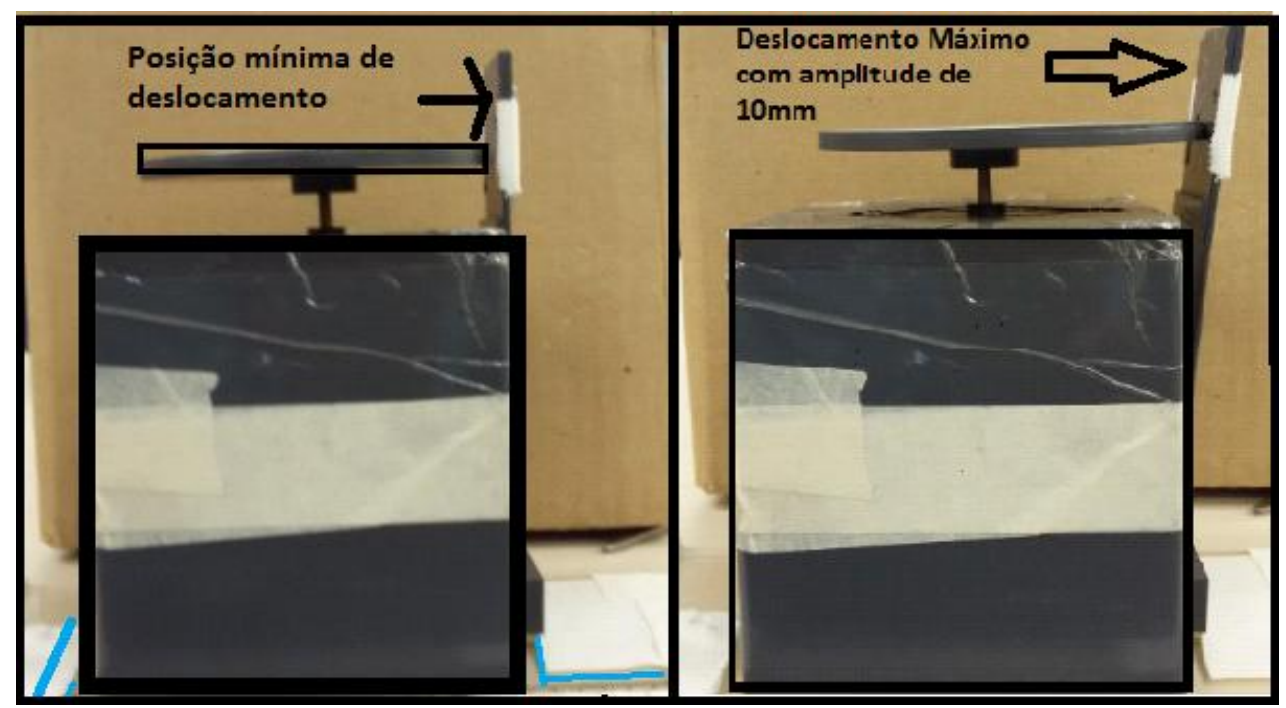

O detector utilizado para obter o valor de dose no alvo foi a câmara de ionização do tipo pinpoint, de volume sensível de $0,015 \mathrm{~cm}^{3}$ (modelo 31014, fabricante PTW Unidos E). A irradiação da mesma foi feita com o gantry do acelerador linear na posição de $0^{0}$, tamanho de campo 10 x 10 $\mathrm{cm}^{2}$, distância fonte-superfície de $100 \mathrm{~cm}$, e à profundidade de $10 \mathrm{~cm}$. O valor da dose de calibração foi de 600 cGy, correspondente a uma fração de SBRT de fígado. O valor do fator de calibração da câmara, que é referenciado em uma instituição de padrão terciário (Instituto Nacional de Câncer) obtida por intercomparação com uma câmara de ionização de outro modelo (Farmer - PTW 30001) referenciada em uma instituição de padrão secundário (Instituto de Radioproteção e Dosimetria), é de 2,349 Gy/nC. As incertezas das medidas estão associadas à resolução digital do eletrômetro utilizado para demonstrar as leituras realizadas na câmara PTW 31014, e ao posicionamento da mesma.

O volume sensível da câmara de ionização foi considerado como o alvo do tratamento e os TLDs como os órgãos de risco. As irradiações foram realizadas no acelerador linear Trilogy®, pertencente ao Hospital do Câncer I, do Instituto Nacional do Câncer (HC I - INCA). O sistema de planejamento (do inglês, Treatment Planning System - TPS) utilizado foi o Eclipse ${ }$. O algoritmo de cálculo utilizado por este sistema de planejamento é o Analytical Anisotropic Algorithm (AAA). Foi utilizada uma energia para o feixe de raios X de $10 \mathrm{MV}$, de acordo com o protocolo de SBRT para fígado [5]. A prescrição de dose para o volume alvo foi de 600 cGy por fração. O simulador, 
com a câmara e os TLDs posicionados nas suas respectivas entradas, foi irradiado com a técnica de irradiação volumétrica em arco (Volumetric Modulated Arc Therapy - VMAT), com as unidades monitoras fornecidas pelo TPS. Tanto a região dos TLDs, quanto a do volume sensível da câmara de ionização, foram contornados no sistema de planejamento, obtendo um planejamento em dois arcos.

Foram obtidas 5 medidas com a câmara de ionização parada, em uma posição situada a uma mesma distância entre as amplitudes que representam a máxima inspiração e expiração, e 5 com a câmara em movimento, onde o acionamento do dispositivo, movido à bateria, foi feito de maneira remota, antes do início da irradiação. O monitor de uma câmera da sala de tratamento possibilitou a visualização do dispositivo para um acionamento do feixe de radiação, de forma manual, no momento em que precede o movimento da câmara pelo mesmo. Com o fator de calibração da câmara utilizada, e o valor médio das medidas obtidas nos dois casos, foi possível então determinar os respectivos valores médios da dose, para avaliar a influência da movimentação do alvo na entrega da dose (efeito interplay).

O intervalo de dose de prescrição sugerido no protocolo de SBRT para fígado, está entre 27,5 e 50,0 Gy, dividido em cinco frações. Neste caso, foram utilizados 30 Gy como dose de prescrição, de modo que cinco frações de 3 Gy foram realizadas com a câmara de ionização parada, e as outras cinco frações de 3 Gy, com a câmara de ionização em movimento, totalizando os 30 Gy de dose prescrita. Foi feito isto com o intuito de comparar a dose medida com a câmara, com a dose registrada pelo sistema de planejamento. Em cada fração administrada, a técnica de irradiação em arco (RapidArc $®)$ foi utilizada.

Foram utilizados 5 TLDs para obter os valores de dose pontual, em duas diferentes regiões referentes a cada rim, e uma na posição relativa à medula. Tais valores foram comparados com os valores obtidos no sistema de planejamento do tratamento. 


\section{RESULTADOS E DISCUSSÕES}

A análise do efeito interplay (Tabela 1), foi realizada pela obtenção da razão entre a dose média adquirida com a câmara parada e a dose média obtida com a câmara em movimento. A diferença percentual em dose foi de 1,3\%, que está em concordância com os valores da literatura [6] e que podemos considerar não significante em relação aos benefícios da entrega de dose com a técnica de VMAT.

Tabela 1: Análise do efeito interplay na dose do alvo.

\begin{tabular}{ccccc} 
& $\begin{array}{c}\text { Valor médio da } \\
\text { resposta da } \\
\text { câmara }(\mathbf{n C})\end{array}$ & $\begin{array}{c}\text { Fator de } \\
\text { calibração da } \\
\text { câmara } \\
(\mathbf{c G y} / \mathbf{n C})\end{array}$ & $\begin{array}{c}\text { Dose média } \\
\text { correspondente } \\
(\mathbf{c G y})\end{array}$ & $\begin{array}{c}\text { Diferença } \\
\text { relativa das } \\
\text { doses médias } \\
\text { obtidas }(\%)\end{array}$ \\
\hline Alvo parado & $2,708 \pm 0,001$ & & $620,000 \pm 12,400$ & $(2 \%)$ \\
Alvo em & $(0,040 \%)$ & $229,000 \pm$ & & $1,300 \pm 0,005$ \\
movimento & $2,675 \pm 0,008$ & $4,580(2 \%)$ & $612,000 \pm 12,240$ & \\
& $(0,300 \%)$ & & $(2 \%)$ & \\
\hline
\end{tabular}

O valor da dose média obtida, mais a sua incerteza, com a câmara parada $(620,000 \pm 12,400$ cGy), demonstrou ser compatível com o valor da dose planejada, na mesma posição, e com a sua respectiva incerteza $(625,500 \pm 6,255 \mathrm{cGy})$. A incerteza do planejamento foi obtida pelo desviopadrão dos valores médios de dose fornecidos pelo histograma dose-volume (DVH).

Os resultados para os órgãos de risco demonstraram que não houve diferença significativa entre os valores medidos pelos TLDs (Tabela 2), com os seus respectivos desvios padrão, com os respectivos valores de dose obtidos através do TPS. Foi realizada a análise em dois diferentes pontos relativos à posição de cada rim, um para a região mais anterior do rim e outra para uma região mais posterior. Somente o resultado obtido para a posição da medula apresentou uma não concordância entre o valor medido e o planejado (mesmo levando em conta os desvios padrões), 
uma vez que o volume associado à medula (no planejamento) apresentou uma distribuição de dose mais heterogênea (Figura 4), que é confirmada com o menor valor de dose média entre os TLDs, pois todos apresentam o mesmo volume, o que resultou num aumento da incerteza da leitura do TLD correspondente à posição da medula.

Figura 4: Cobertura da dose, referente ao valor da dose média na posição da medula

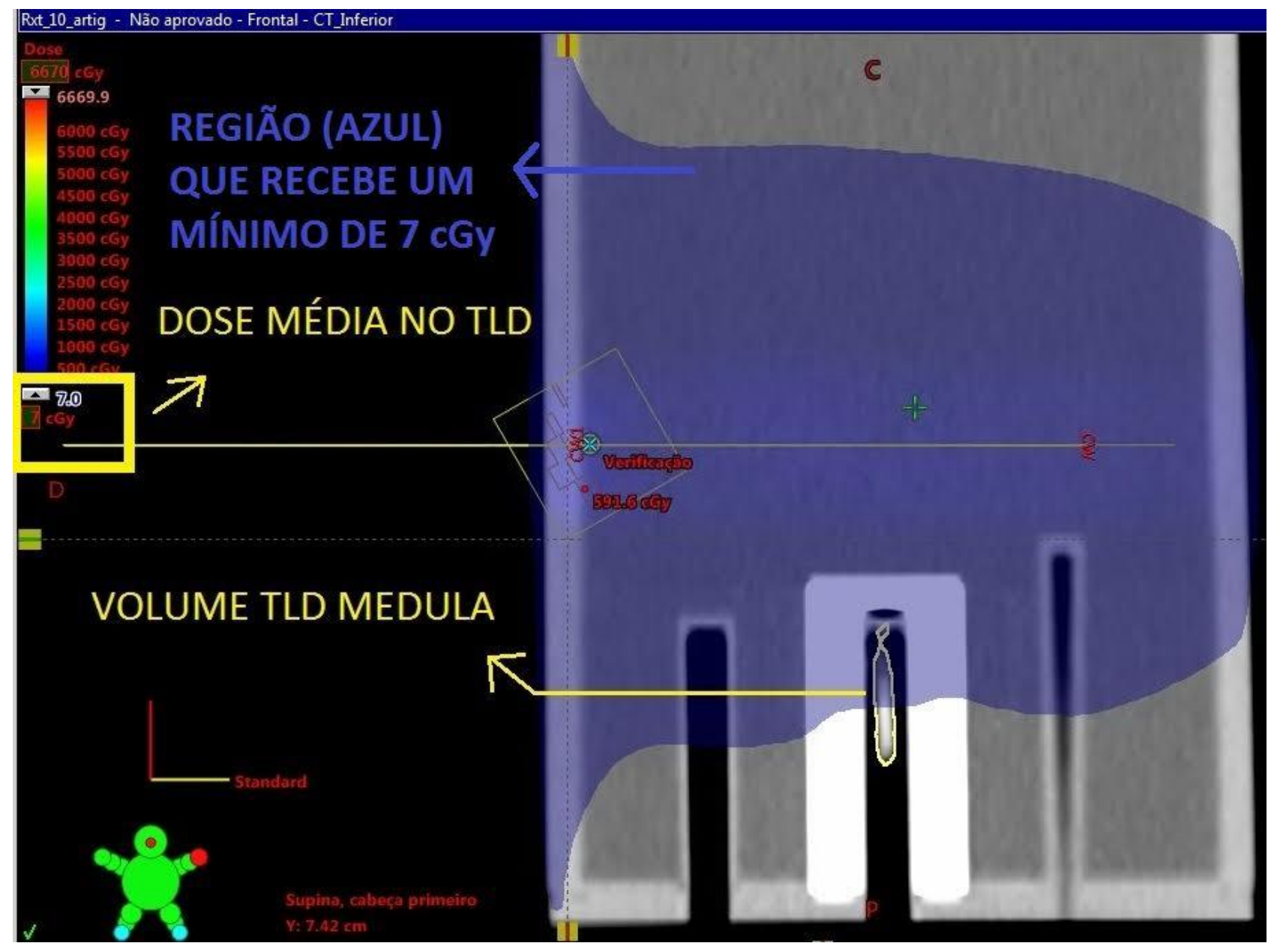


Tabela 2: Valor das doses nos órgãos de risco obtidas pelos TLDs e pelo TPS.

\begin{tabular}{|c|c|c|c|c|}
\hline & $\begin{array}{l}\text { Valor médio das } \\
\text { leituras (ctgs*) }\end{array}$ & $\begin{array}{l}\text { Fator de calibração } \\
\text { dos TLDs (cGy/ctgs) }\end{array}$ & $\begin{array}{c}\text { Dose média } \\
\text { correspondente } \\
\text { (cGy) }\end{array}$ & $\begin{array}{c}\text { Dose média } \\
\text { planejada } \\
\text { (cGy) }\end{array}$ \\
\hline $\begin{array}{c}\mathrm{RE}^{*} \\
\text { (anterior) }\end{array}$ & $\begin{array}{c}2795,0 \pm 111,8 \\
(4 \%)\end{array}$ & & $12,9 \pm 0,5$ & $10,3 \pm 1,8$ \\
\hline $\begin{array}{c}\mathrm{RE} \\
\text { (posterior) }\end{array}$ & $\begin{array}{c}2537,0 \pm 101,5 \\
(4 \%)\end{array}$ & & $11,7 \pm 0,5$ & $9,1 \pm 1,7$ \\
\hline $\begin{array}{c}\mathrm{RD}^{*} \\
\text { (anterior) }\end{array}$ & $\begin{array}{c}3001,0 \pm 180,1 \\
(6 \%)\end{array}$ & $\begin{array}{c}0,00460 \pm 0,00003 \\
(0,6000 \%)\end{array}$ & $13,8 \pm 0,8$ & $13,3 \pm 3,3$ \\
\hline $\begin{array}{c}\mathrm{RD} \\
\text { (posterior) }\end{array}$ & $\begin{array}{c}2933,0 \pm 205,3 \\
(7 \%)\end{array}$ & & $13,5 \pm 0,9$ & $13,6 \pm 5,0$ \\
\hline Medula & $\begin{array}{c}2126,0 \pm 85,0 \\
(4 \%)\end{array}$ & & $9,8 \pm 0,4$ & $6,7 \pm 1,6$ \\
\hline
\end{tabular}

* (ctgs) - contagens; RD - Rim direito; RE - Rim esquerdo.

\section{CONCLUSÕES}

Em virtude do baixo valor na diferença percentual entre as doses com a câmara parada e a câmara em movimento, o efeito interplay foi considerado de menor importância para a técnica de VMAT.

Os resultados do teste end-to-end mostraram que o simulador pode ter utilidade promissora para o controle de qualidade de tratamentos de SBRT de fígado. Novas medidas serão realizadas para assegurar uma maior precisão nos valores das doses, em cada órgão de risco. 


\section{REFERÊNCIAS}

[1] CAPCA Quality Control Standards: Stereotactic Radiosurgery/Radiotherapy. Canadian Association of Provincial Cancer Agencies (CAPCA); 2006. Standards for quality control at Canadian radiation treatment centres.

[2] Potters L, Kavanagh B, Galvin JM. American Society for Therapeutic Radiology and Oncology (ASTRO) and American College of Radiology (ACR) practice guideline for the performance of stereotactic body radiation therapy. Int J Radiat Oncol Biol Phys. 2010;76:326-332.

[3] Benedict SH, Yenice KM, Followill D. Stereotactic Body Radiation Therapy: The Report of AAPM Task Group 101. Med Phys. 2010;37:4078-4101.

[4] Katz A, Winter K, Dawson L, et al. RTOG 0438: A phase I trial of highly conformal radiation therapy for patients with liver metástases.

[5] RTOG 1112 protocol information: Randomized Phase III Study of Sorafenib versus Stereotactic Body Radiation Therapy followed by Sorafenib in Hepatocellular Carcinoma. 2013.

[6] MEYER, J., SCHEFTER, T. Radiation Therapy for Liver Tumors. 288 p. 2017. 